University of Rhode Island

DigitalCommons@URI

6-15-1986

\title{
Polytypism: A Controlled Thermodynamic Phenomenon
}

Shmuel Mardix

University of Rhode Island, mardix@uri.edu

Follow this and additional works at: https://digitalcommons.uri.edu/egr_past_depts_facpubs

Terms of Use

All rights reserved under copyright.

\section{Citation/Publisher Attribution}

Mardix, S. (1986). Polytypism: A controlled thermodynamic phenomenon. Physical Review B, 33(12), 8677-8684. doi: 10.1103/PhysRevB.33.8677

Available at: http://dx.doi.org/10.1103/PhysRevB.33.8677

This Article is brought to you for free and open access by the College of Engineering at DigitalCommons@URI. It has been accepted for inclusion in Past Departments Faculty Publications (CEGR) by an authorized administrator of DigitalCommons@URI. For more information, please contact digitalcommons-group@uri.edu. 


\title{
Polytypism: A controlled thermodynamic phenomenon
}

\author{
S. Mardix \\ Department of Electrical Engineering, University of Rhode Island, Kingston, Rhode Island 02881-0805
}

(Received 25 March 1985)

\begin{abstract}
All ZnS polytypes identified so far are listed; their Zhdanov elements show some peculiar statistical characteristics, which are attributed to their formation mechanism. During the cooling down of the crystals hexagonal domains become less and less stable while the stability of cubic domains increases. Transformations take place by the motion of Shockley partials across the basal planes which, due to the existence of screw dislocations, form an interleaved set of helical surfaces. The characteristics of the Zhdanov elements are explained in view of the above mechanism which also accounts for a number of other reported phenomena. Schneer's theory of polytype formation is discussed.
\end{abstract}

\section{INTRODUCTION}

Polytypism is a phenomenon whereby a compound crystallizes in a variety of periodic layered structures, namely polytypes. The layers comprising a polytype are structurally identical; they can commonly occupy a number of lateral positions relative to their neighboring layers; e.g., the three positions $A, B$, or $C$ which a layer of spheres can occupy in the closest packing of spheres structure. Indeed, the structure of most of the polytypic substances is based on the above closest packing. The various polytypes of a specific substance differ in the number or stacking order of the layers in their unit cell.

The existence of a large number of modifications of the same substance (there are about 200 identified polytypes of $\mathrm{ZnS}$ ) is an uncommon phenomenon presenting two puzzling aspects: (a) structure periodicities with periods of up to a few hundred angstroms, commonly found in organic materials but rarely in minerals; (b) the existence of different stacking sequences in polytypes with the same period.

Theories put forward so far to explain polytypism (see, e.g., the concise review by Baronnet ${ }^{1}$ ) adopt two seemingly opposing approaches: the "thermodynamic theories" attempt to explain the formation of polytypes, both layer arrangement and periodicity, strictly by thermodynamic parameters contributing to the free energy of the structure and completely ignore the possible role of dislocations in the formation mechanism. On the other hand, the "dislocation theories" accept the existence of a few basic structures as stable thermodynamic phases which, due to the interaction of various dislocations, form the multitude of polytypes. The periodic nature of the polytypes, which is the weak part of the thermodynamic theories, is easily explained by the inherent periodicity associated with screw dislocations. The dislocation theories have, however, serious deficiencies in their explanation of the formation of the specific layer sequences experimentally found.

It is the purpose of this article to advance an approach which views the formation of polytypes as a thermodynamic phenomenon taking place with the help of and restrictions imposed by dislocations. This article deals specifically with polytypism in $\mathrm{ZnS}$. We shall present a statistical analysis of the currently identified polytypes and show that their stacking sequences are formed as a result of temperature-dependent stability characteristics of hexagonal and cubic groups of layers. The process is assisted by Shockley partial dislocations and controlled by the topology imposed on the crystal by screw dislocations.

\section{OBSERVED STACKING SEQUENCES: STATISTICAL CHARACTERISTICS}

The statistical characteristics described in this section are based on an analysis of all $\mathrm{ZnS}$ polytypes identified so far: the population. The elementary stacking sequences of these polytypes are presented in Table I. The unit cell of polytypes belonging to the space groups $P 3 m 1\left(C_{3 v}^{1}\right)$ and $P 6_{3} m c\left(C_{6 v}^{4}\right)$ coincides with their elementary stacking sequence, while the unit cell of $R 3 \mathrm{~m}\left(C_{3 v}^{5}\right)$ polytypes consists of three consecutive elementary stacking sequences and thus includes $3 N$ layers, where $N$ is the number of layers in the elementary stacking sequence.

The Zhdanov symbol ${ }^{2}$ is most commonly used to represent a stacking sequence; the symbol consists of an ordered set of elements $\left(Z_{1}, Z_{2}, \ldots, Z_{k}, \ldots, Z_{m}\right)$. Each element $Z_{k}$ represents a sequence of one hexagonally stacked layer (such as $B$ in $A B A$ ) followed by $Z_{k}-1$ cubically stacked layers (such as $B$ in $A B C$ ). For periodic stacking sequences $m$ must be even. The presentation of a polytype by its Zhdanov symbol is not unique, a number of symbols may represent the same polytype, e.g., the ten layered polytype (4213) can also be represented by (3 124), (2134), (2431), and a few more.

Two equivalent symbols are composed of the same sequence of elements in either the same direction such as (4213) and (2134) or in opposite directions such as $\left(\begin{array}{llll}4 & 2 & 1 & 3\end{array}\right)$ and $\left(\begin{array}{llll}3 & 1 & 2 & 4\end{array}\right)$.

In order to facilitate reference to the table a uniform presentation of the stacking sequences by their Zhdanov elements was adopted: The Zhdanov symbol of a polytype is considered as a base- $N$ number; from all equivalent symbols which may represent a polytype the one with the highest value is selected. This selection assigns a unique 
symbol to each polytype and can also be used to define an order between polytypes with a given value of $N$.

The polytypes in Table $I$ are arranged according to the number of layers in their elementary stacking sequence and the value of this sequence. The Zhdanov symbol is preceded by the number of layers in the unit cell and the space-group symbol: $L$ for $P 3 m 1, H$ for $P 6_{3} m c$ and $R$ for $R 3 \mathrm{~m}$.

As an example let us look at the polytype (42 213 ) mentioned above: the value associated with this presentation is the base-10 number 4213. The values of the other possible presentations are $2134,1342,3421,3124,1243,2431$, and 4312 . Since the highest value is 4312 , the polytype will be assigned the symbol $30 R(4312)$.

A statistical analysis of the Zhdanov elements shows some peculiar distribution characteristics.

(a) There is a total of 808 Zhdanov elements $Z$ in the population of 194 polytypes, 778 of them have values in the range of $1 \leq Z \leq 15$; their distribution is presented in Fig. 1 (the remaining 30 elements have values in the range of $16 \leq Z \leq 50$ ). The distribution function in Fig. 1 appears to be composed of two separate sequences, one of the even and the other of the odd $Z$ elements. Furthermore, if $N_{z}$ denotes the number of elements with a value of $Z$, then each of the sequences $N_{2 k}$ and $N_{2 k+1}$, $k=1,2, \ldots, 7$ constitutes a geometric sequence of the type $a_{n}=a_{0}\left(\frac{1}{2}\right)^{n}$. Assuming this relation holds for the sequence of even $Z$ values, $N_{2 k}$, one can get an approximate value for $a_{0}$ from the relation $a_{0} \approx \sum_{k=1}^{7} N_{2 k}$. The value of $a_{0}$ is thus $a_{0}=286$. The computed values of the sequence $N_{2 k}=286\left(\frac{1}{2}\right)^{k}, k=1,2, \ldots, 7$ are given in Fig. 1, joined by the continuous curve $N_{z}=286\left(\frac{1}{2}\right)^{Z / 2}$.

In a similar way one gets $N_{2 k+1}=465\left(\frac{1}{2}\right)^{k}$,

TABLE I. The elementary stacking sequences of all currently identified $\mathrm{ZnS}$ polytypes other than the cubic and hexagonal structures. The Zhdanov symbol is preceded by the number of layers in the unit cell and the space-group symbol: $L$ for $P 3 m 1$ ( $C_{3 v}^{1}$ ), $H$ for $P 6_{3} m c\left(C_{6 v}^{4}\right)$, and $R$ for $R 3 m\left(C_{3 v}^{5}\right)$.

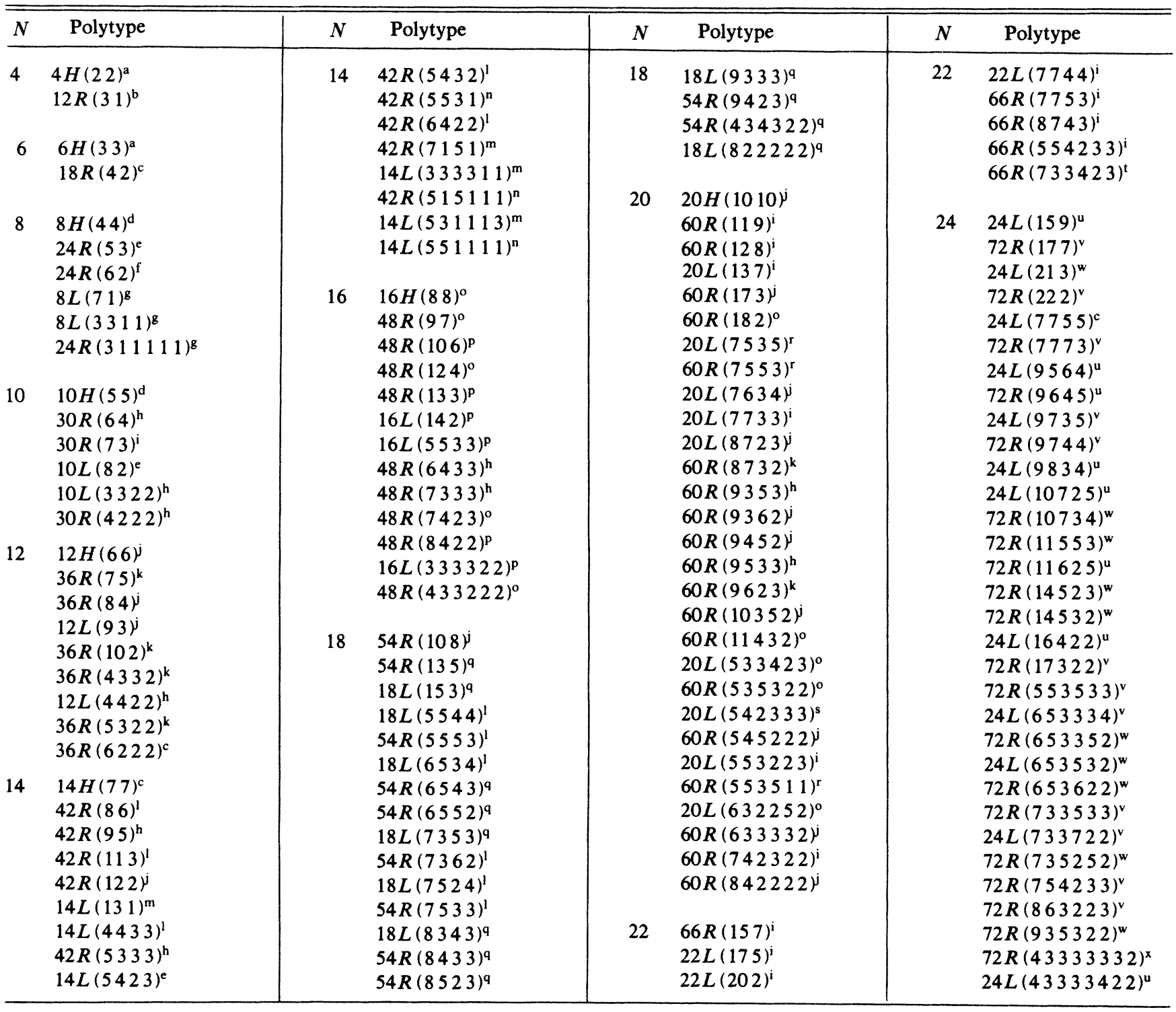


TABLE I. (Continued).

\begin{tabular}{|c|c|c|c|c|c|c|c|}
\hline$N$ & Polytype & $N$ & Polytype & $N$ & Polytype & $N$ & Polytype \\
\hline 24 & 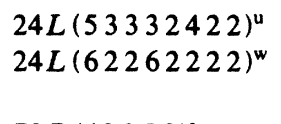 & 28 & $\begin{array}{l}28 L(262)^{\mathrm{y}} \\
28 L(9955)^{\mathrm{e}} \\
84 R(11845)^{\mathrm{j}}\end{array}$ & 32 & $\begin{array}{l}96 R(17564)^{\mathrm{aa}} \\
32 L(1372343)^{\mathrm{g}}\end{array}$ & 38 & $\begin{array}{l}114 R(21962)^{j} \\
114 R(135226262)^{j}\end{array}$ \\
\hline 26 & $\begin{array}{l}78 R(10853)^{\mathrm{s}} \\
78 R(13553)^{\mathrm{i}} \\
78 R(17342)^{\mathrm{i}} \\
26 L(17423)^{\mathrm{e}} \\
26 L(753335)^{\mathrm{s}} \\
26 L(753533)^{\mathrm{s}}\end{array}$ & & $\begin{array}{l}28 L(13573)^{\mathrm{y}} \\
84 R(13735)^{\mathrm{y}} \\
28 L(18352)^{\mathrm{y}} \\
28 L(21322)^{\mathrm{y}} \\
84 R(55533322)^{\mathrm{z}} \\
84 R(73333333)^{\mathrm{y}}\end{array}$ & 34 & $\begin{array}{l}102 R(313)^{\mathrm{aa}} \\
34 L(75355522)^{\mathrm{aa}}\end{array}$ & 40 & $\begin{array}{l}40 L(11117452)^{\mathrm{s}} \\
120 R(11118352)^{\mathrm{s}} \\
120 R(13511533)^{\mathrm{aa}} \\
120 R(2932222)^{\mathrm{aa}}\end{array}$ \\
\hline & $26 L(773333)^{i}$ & & $28 L(3333333322)^{y}$ & & $36 L(23373)^{\mathrm{g}}$ & 44 & $44 L(377)^{j}$ \\
\hline & $78 R(773342)^{i}$ & & & & $\begin{array}{l}36 L(1155555)^{\mathrm{s}} \\
108 R(75555522)^{\mathrm{s}}\end{array}$ & & $44 L(176174)^{\mathrm{j}}$ \\
\hline & $78 R(843533)^{\mathrm{s}}$ & 30 & $90 R(18372)^{\text {aa }}$ & & $108 R(115335243)^{s}$ & & \\
\hline & $\begin{array}{l}26 L(8533325)^{\mathrm{s}} \\
78 R(935333)^{\mathrm{i}}\end{array}$ & & $\begin{array}{l}90 R(775524)^{\mathrm{s}} \\
90 R(863553)^{\mathrm{aa}}\end{array}$ & & & 54 & $162 R(504)^{\mathrm{s}}$ \\
\hline 28 & $\begin{array}{l}28 L(235)^{\mathrm{j}} \\
84 R(253)^{\mathrm{j}}\end{array}$ & 32 & $\begin{array}{l}96 R(13955)^{g} \\
32 L(14855)^{8}\end{array}$ & 38 & $\begin{array}{l}114 R(299)^{j} \\
114 R(353)^{j}\end{array}$ & 64 & $64 L(455113)^{\mathrm{s}}$ \\
\hline \multicolumn{2}{|c|}{$\begin{array}{l}{ }^{\mathrm{a}} \text { Reference } 21 . \\
{ }^{\mathrm{b}} \text { Reference } 22 . \\
{ }^{\mathrm{c}} \text { Reference } 23 . \\
{ }^{\mathrm{d}} \text { Reference } 24 . \\
{ }^{\mathrm{e}} \text { Reference } 25 . \\
{ }^{\mathrm{f}} \text { Reference } 6 . \\
{ }^{\mathrm{g}} \text { Reference } 26 .\end{array}$} & \multicolumn{2}{|c|}{$\begin{array}{l}{ }^{\mathrm{h}} \text { Reference } 27 . \\
{ }^{\mathrm{i}} \text { Reference } 28 . \\
{ }^{\mathrm{j}} \text { Reference } 29 . \\
\mathrm{k} \text { Reference } 17 . \\
{ }^{\mathrm{I}} \text { Reference } 30 . \\
{ }^{\mathrm{m}} \text { Reference } 31 . \\
{ }^{\mathrm{n}} \text { Reference } 32 .\end{array}$} & \multicolumn{2}{|c|}{$\begin{array}{l}{ }^{\mathrm{O}} \text { Reference } 4 . \\
{ }^{\mathrm{p}} \text { Reference } 33 . \\
{ }^{\mathrm{q}} \text { Reference } 34 . \\
{ }^{\mathrm{r}} \text { Reference } 18 . \\
\text { s Reference } 35 . \\
{ }^{\mathrm{t}} \text { Reference } 3 . \\
{ }^{\mathrm{u}} \text { Reference } 36 .\end{array}$} & \multicolumn{2}{|c|}{$\begin{array}{l}{ }^{\mathrm{v}} \text { Reference } 37 . \\
{ }^{\mathrm{w}} \text { Reference } 38 . \\
{ }^{\mathrm{x}} \text { Reference } 39 . \\
{ }^{\mathrm{y}} \text { Reference } 40 . \\
{ }^{\mathrm{z}} \text { Reference } 41 . \\
{ }^{\text {aa }} \text { Reference } 42 .\end{array}$} \\
\hline
\end{tabular}

$k=1,2, \ldots, 7 \quad\left(\sum_{k=1}^{7} N_{2 k+1}=465\right)$. The computed values of this sequence are given in Fig. 1, joined by the continuous curve $N_{z}=465\left(\frac{1}{2}\right)^{(Z-1) / 2}$. The number of occurrences of Zhdanov elements with $Z=1$ does not fit this curve; this is an important property and will be discussed in detail.

It should be noted that no adjustable parameters appear in the theoretical expressions for the number of elements in the distribution sequences of the even and odd Zhdanov elements. The scaling factor $a_{0}$ was computed from the experimental values of $N_{2 k}$ and $N_{2 k+1}, k=1,2, \ldots, 7$.

(b) Groups of Zhdanov elements of the type "3Z 3." The importance of this specific group will be clarified in the discussion. The value of $Z$ is even, specifically 4 , in only 3 out of the 49 groups (6\%) of the type " $3 Z 3$ " found in the population. The polytypes with an even value of $Z$ are $18 L(8343), 54 R(434322)$, and $32 L(1372343)$. It is interesting to note that the first two polytypes were found in the same specimen and that the first one was found in two different parts of the specimen. This implies that three out of the four known polytypic regions found to include the group " 343 " belong to the same specimen.

(c) Neighboring hexagonal layers appear as 1's in the Zhdanov symbol. Polytypes with neighboring hexagonal layers in their stacking sequence constitute a subset of the general population with the following characteristics: (1) Only 12 polytypes out of the population of 194 have one or more of their Zhdanov elements equal to 1 . (2) There are no even elements in this subset.

\section{THE ROLE OF DISLOCATIONS IN $\mathrm{ZnS}$ POLYTYPE FORMATION}

A large volume of experimental evidence establishing the role of dislocations in the mechanism of $\mathrm{ZnS}$ polytype formation is presented in the literature. ${ }^{3-9}$ The elements of this mechanism are summarized below. ${ }^{9}$

$\mathrm{ZnS}$ has two thermodynamically stable phases: the hexagonal $(2 H)$ phase is the high-temperature modification (above about $1300 \mathrm{~K}$ ) while the cubic phase is the stable modification at room temperature. ${ }^{10,11}$

Almost all available information on polytypism in $\mathrm{ZnS}$ is associated with vapor-phase-grown crystals. The crystals grow at a temperature of about $1400 \mathrm{~K}$. A substrate of polycrystalline $\mathrm{ZnS}$ is first formed on the walls of the growth tube. Hexagonal $2 H$ whiskers start to grow out of the substrate; the length of the whiskers coincides with their $c$ axis. At a certain, yet unknown stage, screw dislocations are formed and the $2 H$ structures continue to grow around them. For continued growth around a screw dislocation its Burgers vector $b$ must be equal to an integral multiple of the $2 H$ unit cell or $|\mathbf{b}|=2 n c_{0}$ where $n$ is an integer and $c_{0}$ denotes the interlayer distance along the $c$ axis. As a consequence of the strain surrounding the screw dislocation, a hollow tube starts to develop around the dislocation line. In that part of the crystal from which the core of the dislocation is removed the topology of the basal planes is that of a system of $2 n$ interleaved helical surfaces having a pitch equal to $|\mathbf{b}|$.

Shockley partials with a Burgers vector $\mathbf{p}=\left(\mathbf{a}_{j}-\mathbf{a}_{k}\right) / 3$ 


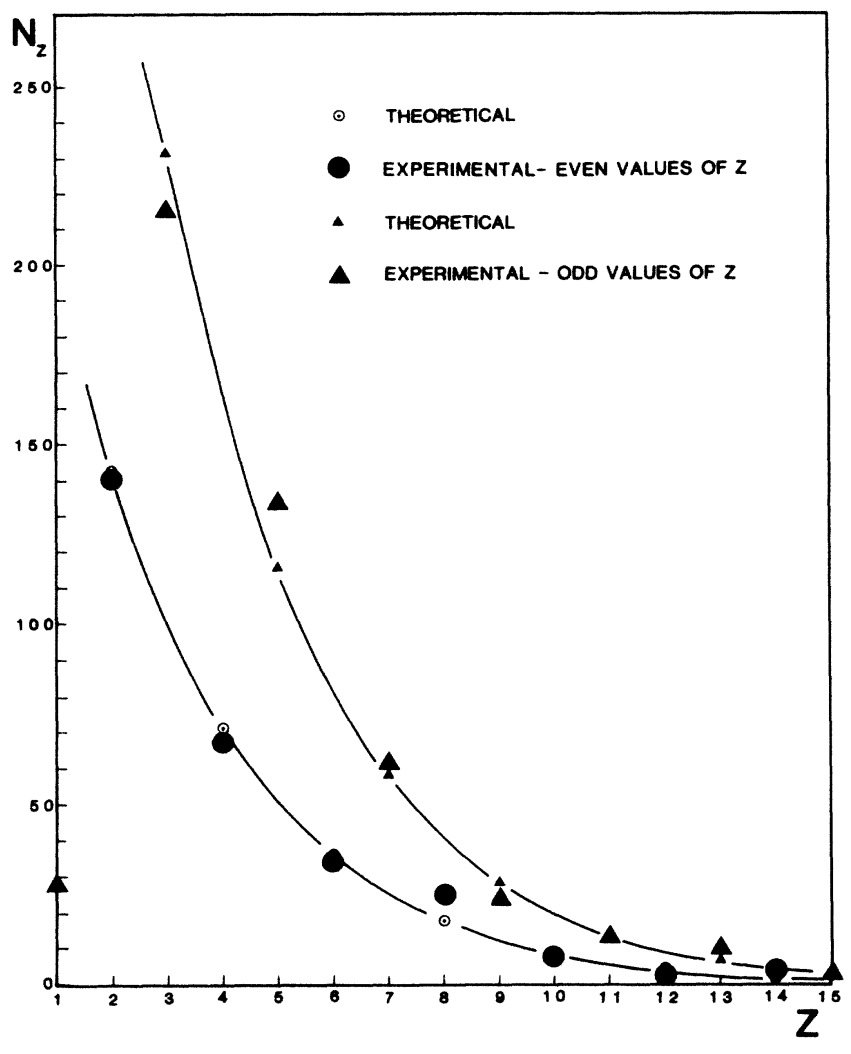

FIG. 1. Distribution of the Zhdanov elements of the known $\mathrm{ZnS}$ polytypes. $N_{\mathrm{z}}$ is the number of elements having a value of $Z$. Experimental values of $N_{z}$ are separated into two groups: those belonging to even $Z$ values (solid circles) and those belonging to odd $Z$ values (solid triangles). The experimental values are connected by the two curves $N_{z}=286\left(\frac{1}{2}\right)^{z / 2}$ and $N_{z}=465\left(\frac{1}{2}\right)^{(z-1) / 2}$ for the even and odd $Z$ values, respectively. The computed $N_{z}$ values for integral values of $Z$ are given as open circles and triangles.

$(j \neq k=1,2,3)$, where $\mathbf{a}_{j}$ and $\mathbf{a}_{k}$ are two basal plane unit vectors, can glide across the basal planes of the crystal to produce two effects.

(a) One part of the crystal slips in respect to the other. The slip is given by the slip vector $\mathbf{s}$ where $\mathbf{s}=\mathbf{p}$.

(b) The two neighboring layers on the two slipped parts of the crystal will find themselves in a new environment, a hexagonal layer will become cubically stacked and vice versa; all other basal planes in the crystal will not be structurally affected by the motion of the partials and the associated slip.

The formation of polytypes in $\mathrm{ZnS}$ is a result of solidstate transformations of the $2 H$ high-temperature phase. The volume surrounding the screw dislocation line in those regions where the core of the dislocation is not hollow is highly strained and basal-plane partials can easily be formed. As the crystals cool down from the growth temperature the $2 \mathrm{H}$ phase becomes unstable and the partial dislocations start to move across the basal planes transforming the stacking sequence into a more stable one. Due to the helical configuration of the basal planes a partial dislocation will move across consecutive planes, positioned along the $c$ axis at a distance equal to the mag- nitude of the Burgers vector $b$. This periodic layer transformation results in a polytype with an elementary stacking sequence having $N$ layers so that $N=|\mathbf{b}| / c_{0}$.

Polytypic regions with macroscopic dimensions along the $c$ axis $(\geq 50 \mu \mathrm{m})$ can be formed only if the dislocation density in the region is small and only if the region is unstrained so as to enable the Shockley partials to move across the basal planes without being pinned. These conditions are met in those regions where the core of the screw dislocation is hollow; Shockley partials, moving from the neighboring strained regions, can climb unimpeded to transform the structure into a perfect macroscopic polytypic region.

In summary, the Shockley partial dislocations are the means by which structure transformations are taking place while the screw dislocation imposes its periodicity on the transformed structure.

\section{DOMAINS AND THEIR STABILITY}

While the dislocation mechanism can account for the periodicities of the polytypes, it is nonselective as far as the stacking sequence is concerned; any combination of Shockley partials will interact with the screw dislocation to create a polytype. The characteristics of the identified stacking sequences indicate, however, that the transformations induced by the partials are not taking place randomly; specific selection rules seem to govern the formation and motion of these partials. We shall show that a few rules, thermodynamic in nature, can account for the observed characteristics of the stacking sequences as well as for some other phenomena reported in the literature.

A "domain" is defined here as a group of basal-plane layers of the same type, either hexagonal or cubic, bounded by layers of the other type, $n_{h}$ will denote a domain consisting of $n$ hexagonally stacked layers, bounded by cubic layers on both ends of the stack. In a similar way $n_{c}$ will denote an $n$-layered cubic domain. A polytype can be considered as a periodic arrangement of alternate hexagonal and cubic domains. A group of $m$ 1's in the Zhdanov symbol represents the $(m+1)_{h}$ domain. The Zhdanov element $Z>1$ represents the domain $(Z-1)_{c}$. For example, the polytype $12 L(8112)$ is a periodic sequence of the domains $1_{h} 7_{c} 3_{h} 1_{c}$. The same polytype will appear in Jagodzinski's notation ${ }^{12}$ as " hcccccchhhc," where " $h$ " and " $c$ " denote hexagonal and cubic layers, respectively. It should be noted that the domain notation is simply a shorthand description of Jagodzinski's notation.

The empirical rules governing the stability of domains can now be stated: The stability of hexagonal and cubic domains is a function of temperature and depends on the domain's size. The stability of cubic domains decreases with temperature while the stability of hexagonal domains increases. At each temperature there is a hexagonal domain $m_{h}$ which is the most stable domain in comparison to the other hexagonal ones; the value of $m$ for this most-stable domain increases with increasing temperature. In a similar way the value of $m$ of the most stable cubic domain $m_{c}$ decreases with increasing temperature. This state of affairs can be understood if we assume the tem- 
perature dependence of the structural energy of hexagonal domains to be qualitatively given in Fig. 2(a), where the average energy per layer $E$ for the domains $1_{h}$ and $2_{h}$, in reference to that of the hexagonal $2 H$ phase, is given as a function of temperature. In the temperature range $T<T_{1 h}$ the $1_{h}$ domain is the most stable one; $2_{h}$ is the most stable domain in the range $T_{1 h}<T<T_{2 h}$. We assume that consecutive $n_{h}$ domains will $n>2$ are stable at temperatures $T>T_{2 h}$; the population used for the analysis is, however, too small to enable discrimination between them.

In a manner similar to that of Fig. 2(a), the average energy per layer for cubic domains is given as a function of temperature in Fig. 2(b). The energy per layer of the cubic phase is taken as a reference. The detailed interrelation of the curves of Fig. 2(b) to those of Fig. 2(a) is not yet clear.

\section{THE FORMATION OF THE OBSERVED STACKING SEQUENCES}

It was mentioned above that the various $\mathrm{ZnS}$ polytypes are formed during the cooling down period from the growth temperature. We shall now follow the transformations taking place during this period.

Starting from the growth temperature and cooling down, the crystals reach a temperature where the $2 \mathrm{H}$ structure becomes unstable. We assume that this temperature is higher than $T_{1 c}$ [Fig. 2(b)]; the most stable domain at this temperature range is the $1_{c}$ domain. The only domain, however, which can be created by a Shockley partial in a $2 \mathrm{H}$ structure is the $2_{c}$ domain. $2_{c}$ domains will therefore be created; if the temperature will stay above $T_{1 c}$ for a sufficiently long time they will transform into two $1_{c}$ domains. The transformations taking place can be described in the following way:

$$
\begin{aligned}
\cdots h \underline{h} \underline{h} h h h \cdots & \rightarrow \cdots h h c \underline{c} \underline{h} h h \cdots \\
& \rightarrow \cdots h h c h c h h \cdots .
\end{aligned}
$$

It was mentioned in a previous section that a transformation induced by the motion of a partial dislocation changes the types of two neighboring layers; these are underlined in the above description. Using the Zhdanov notation the transformations will appear as

$$
\cdots 111111 \cdots \rightarrow \cdots 1131 \cdots \rightarrow \cdots 1122 \cdots .
$$

Notice that a pair of 2's are created as Zhdanov elements; this is the only single transformation which can create an even-valued Zhdanov element and be associated with an energy decrease of the system.

Further cooling of the crystals brings them into the stability range of $2_{h}$ and $2_{c}$ domains. The $2_{c}$ domains will not transform further in this temperature range. Also, only $n_{h}$ domains with $n=2$ are stable, and therefore one can expect an increase in the density of $2_{h}$ and $2_{c}$ domains. The pair of $1 c$ domains which were formed in the former stage will not tend to transform back into a $2_{c}$ domain by the possible transformation

$$
\cdots \text { hhch } \underline{c} h h \cdots \rightarrow \cdots \text { hhcchhh } \cdots \text {, }
$$

while the creation of the $2_{c}$ domain will result in an energy loss, the associated transformation $2_{h} \rightarrow 3_{h}$ will result in an energy gain.

When the crystals cool down to temperatures below $T_{1 h}$ and $T_{2 c}$ they reach the final transformation stage. At this stage $2 h$ domains and larger hexagonal domains which were not yet transformed will tend to transform in such a way as to increase the cubic domains and either completely disappear or leave behind $1_{h}$ domains. As an example, the stacking sequence $\cdots c c \underline{h} \underline{h} c c \cdots$ will transform to $\cdots \operatorname{ccccc} \cdots$, while $\cdots \operatorname{cchhh} \cdot \cdots$ will transform to either $\cdots \operatorname{ccch} c \cdots$ or $\cdots$ cchccc $\cdots$ and all the above transformed regions will stay as stable stacking sequences.

Notice that the end result of the transformations in this stage is an increase by two of Zhdanov elements. This means that even Zhdanov elements will increase but will stay even while odd elements will stay odd. This explains the breakdown of the Zhdanov elements in the population into the two groups: the sequence of the even elements and that of the odd elements.

Let us now follow the transformations of a single cubic domain, say $3_{c}(\cdots h h h h c c c h h h h \cdots)$. There are four possible transformations which will determine if the domain will be transformed into a larger one or stay unchanged:
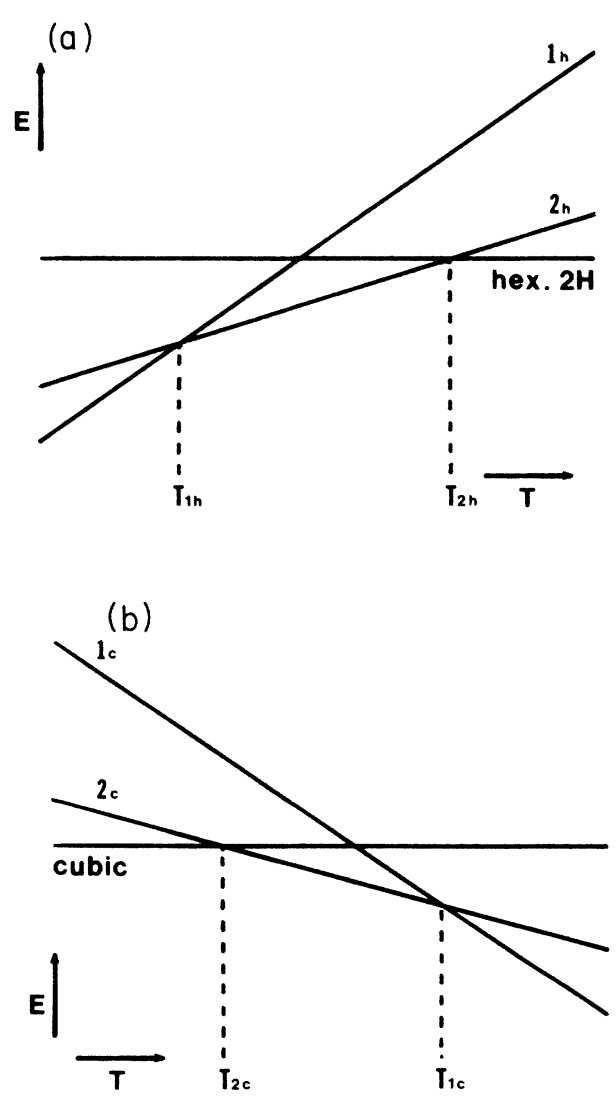

FIG. 2. Average energy per layer for specific domains as a function of temperature. (a) Hexagonal domains $1_{h}$ and $2_{h}$, in reference to the hexagonal $2 H$ structure. (b) Cubic domains $1_{c}$ and $2_{c}$ in reference to the cubic phase. 
(a) $\cdots h h \underline{h} \underline{h} \operatorname{ccchhhh} \cdots \rightarrow \cdots$ hhccccchhhh $\cdots$,

(b) $\cdots$ hhhhccch $\underline{h} h \boldsymbol{W} \cdots \rightarrow \cdots$ hhhhccccchh $\cdots$,

(c) $\cdots h \underline{h} \underline{h} h c c c h h h h \cdots \rightarrow \cdots$ hchccchhhh $\cdots$,

(d) $\cdots$ hhhhccch $\underline{h} \underline{h} h \cdots \rightarrow \cdots$ hhhhccchcch $\cdots$.

From these four transformations (a) and (b) are mutually independent and so are (c) and (d); (a) and (c) are mutually exclusive; the same applies to (b) and (d). From the four possible combinations of transformations the combination (c)/(d) [(c) followed by a type (d) transformation] will preserve the original cubic domain as there are no further transformations which can affect it. The other three possible combinations of transformations will result in a transformed domain: (a) $/($ b) will give a $7 c$ domain while (a) $/($ c) and (b)/(d) will each result in a $5 c$ domain. Assuming equal probability for each of the transformations (a)-(d), one concludes that the probability of a single cubic domain to transform is $3 / 4$. For a cubic domain bounded on one side by a $1_{h}$ domain and on the other side by a larger hexagonal domain (1's on one side and no 1's on the other in the Zhdanov notation), only (a) or (c) can take place, or, only (b) or (d). In the first case (a) will transform the domain while (c) will preserve it. The same will apply, respectively, to (b) and (d). The probability of the domain to transform will now be $1 / 2$.

Even-layered domains are first formed as a Zhdanov pair " 22, ," and therefore they will satisfy the conditions of the last case all during their sequence of transformations. As a result of an even Zhdanov element having, at each stage, the same probability to transform or to be preserved one would expect the sequence of even elements to satisfy the relation $a_{k}=a_{0}\left(\frac{1}{2}\right)^{k}$, which was experimentally found and presented in Fig. 1.

We mentioned above that the first stage of formation of even $Z$ elements is the formation of a pair of 2's with the most common sequential transformations into larger even elements. There is, however, a possibility, through a combination of transformations, for an even number to convert to an odd one:

\section{$\cdots h \underline{h} \underline{h} h c h c h h h \underline{h} \underline{h} \cdots \rightarrow \cdots h c c h c h c h h \underline{h} \underline{c} c \cdots \rightarrow \cdots h c c h c h c \underline{h} \underline{h} \operatorname{ch} c \cdots \rightarrow \cdots h c c h c h c c c c h c \cdots$}

The combination $\cdots 1112211111 \cdots$ transformed into the combination $\cdots 3252 \cdots$. This last combination includes an even element bounded by two odd elements with one of them greater than 3 . Only a very complex sequence of transformations can lead to an even number bounded by two 3's. The probability for this last configuration to be formed is therefore very small, which explains the rarity of the " $3 Z 3$ " configuration with an even $Z$.

The formation of the sequence of odd-layered domains is similar to that of the sequence of even-layered ones but somewhat more complicated. While even elements are created only as neighboring pairs, the odd elements can be created either in pairs or as single ones. The formation of the " 33 " pairs was detected in stress-induced transformations at room temperature. ${ }^{13}$ As the single elements have a transformation probability of $3 / 4$ in comparison to $1 / 2$ for elements in a pair, one can expect to find less 3's than anticipated from the relation $a_{n}=a_{0}\left(\frac{1}{2}\right)^{n}$. The fact, that the number of 3's in the population was indeed found to be below the theoretical value and that of 5's above (see Fig. 1), may therefore be of significance and not just a statistical aberration.

The small number of 1's (" $h h$ " groups) as elements of the population is a straightforward consequence of their instability through the whole temperature range $T<T_{1 h}$; they can only transform (to cubic domains) without being created. Their existence at room temperature is possibly associated with a rapid cooling of the crystal to room temperature before most of the Shockley partials are able to cover macroscopic distances. However, rapid cooling will also shorten the only time during which even elements can be created, thus practically preventing their formation. The lack of even elements in the above subset of the population is thus explained.

Thirty Zhdanov elements out of the 808 were found to have values of $Z$ larger than 15 , which is somewhat higher than that predicted from Fig. 1. We assumed so far that the four transformations [(a)-(d)] have equal probabilities; however, for transformations taking place at close to room temperature, in the range of high stability for large cubic domains, one can expect (a) and (b) to predominate over (c) and (d). The domains with $Z>15$ may therefore be a result of low-temperature transformations.

\section{DISCUSSION}

Detailed experimental data concerning the $E$ versus $T$ relations for polytypic structures is necessary in order to directly establish the validity of the empirical rules of domain stability assumed in this article. As this information is not available and as there is no current theory which will enable the computation of the above relations, the presented set of rules appears as an ad hoc approach explaining the statistical characteristics of the Zhdanov elements. It is important therefore to find additional supporting evidence. We shall now present some experimental results from the literature and discuss them in terms of domain stability.

Schneer developed a theory of polytypism based on purely thermodynamic considerations of interaction energies between cubic and hexagonal layers. ${ }^{14} \mathrm{He}$ defines a layer distribution function $D$ and derives a relation between $D, n_{h}$, and $n_{c}$, where $n_{h}$ and $n_{c}$ are the number of hexagonal layers and the number of cubic layers, respectively, in the structure. The above relation is given by $(1-D) / D=n_{h} / n_{c}$. Layer interaction energies are defined in terms of the interaction contacts $n_{\mathrm{ch}}, n_{\mathrm{cc}}$, and $n_{\mathrm{hh}}$, where $n_{\mathrm{ch}}$ is the number of contacts between cubic 
layers and hexagonal ones, $n_{\mathrm{cc}}$ is the number of cubiccubic contacts, and $n_{\mathrm{hh}}$ is the number of hexagonalhexagonal contacts. $\phi$ represents the distribution of the interaction contacts and is given by

$$
\phi=\left[n_{\mathrm{ch}}-\left(n_{\mathrm{cc}}+n_{\mathrm{hh}}\right)\right] /\left(n_{\mathrm{ch}}+n_{\mathrm{cc}}+n_{\mathrm{hh}}\right) .
$$

The claim is made that the stacking sequences of polytypes should give a maximum value of $\phi$. This maximum value for any assembly is shown to be $\phi_{\max }=4 D-1$ for $D<\frac{1}{2}$ and $\phi_{\max }=3-4 D$ for $D>\frac{1}{2}$. Schneer presents the $\phi-D$ relation for the then known $\mathrm{SiC}$ polytypes and shows that they follow the theoretical relation for $\phi_{\max }$.

Rai and Krishna ${ }^{15,16}$ repeated Schneer's computations for the $\mathrm{ZnS}, \mathrm{CdI}_{2}, \mathrm{PbI}_{2}$ and $\mathrm{SiC}$ polytypes and found that they also obey the theoretical $\phi-D$ relation. The above correspondence between experiment and theory was claimed to be a verification of the theory.

It can, however, be easily shown that the theoretical relation between $\phi_{\max }$ and $D$ for $D<\frac{1}{2}$ is an identity for polytypes which do not have neighboring cubic layers (no Zhdanov elements greater than 2), while the relation for $D>\frac{1}{2}$ holds as an identity for polytypes which do not have neighboring hexagonal layers (no 1's as Zhdanov elements). This last condition is satisfied for most of the $\mathrm{ZnS}$ polytypes and for all SiC polytypes; the few exceptions to the $\phi-D$ relation given by Rai and Krishna are those ZnS polytypes which do have l's as Zhdanov elements. Almost all quoted $\mathrm{CdI}_{2}$ polytypes do not have neighboring cubic layers; the two exceptions do not lie on the $\phi_{\max }-D$ lines. From the five $\mathrm{PbI}_{2}$ polytypes which were checked, three do not have neighboring cubic layers, one does not have neighboring hexagonal layers, and the fifth which has both cubic and hexagonal neighboring layers does not indeed satisfy the $\phi_{\max }-D$ relation.

The lack of 1's in the Zhdanov sequences of $\mathrm{ZnS}$ and $\mathrm{SiC}$ polytypes should be viewed according to our interpretation as a manifestation of the instability of neighboring hexagonal layers at one of the polytype formation stages. In a similar way the instability of neighboring cubic layers would account for the lack of Zhdanov elements greater than 2 in the stacking sequences of the $\mathrm{CdI}_{2}$ polytypes.

The above discussion should not be taken as a rejection of Schneer's theory; we only claim that its experimental substantiation given so far is invalid. If one could derive the empirical rules brought here from Schneer's theory or from any other thermodynamic theory of polytype formation, then that theory could constitute the theoretical basis for these rules.

The instability of neighboring hexagonal layers at room temperature and their stability at a higher-temperature range can also explain the formation of double-polytype regions. ${ }^{17}$ These regions consist of a large number of very narrow $(<1 \mu \mathrm{m})$ subregions. It was shown in a recent article $^{18}$ that the structure of each subregion is one of the two possible transformation products of a parent polytype which includes a single pair of neighboring hexagonal layers in its elementary stacking sequence. While the crystal cools down to room temperature the hexagonal pairs move out of their stability range and form a supercooled structure. This structure breaks down suddenly into the transformation products in such a way that the " $h h$ " pairs are eliminated and the structure is left with the low-temperature stable single " $h$ " layers.

Our empirical stability rules are also in accord with Baars and Brandt's annealing experiments on $\mathrm{ZnS}$ crystals. ${ }^{19}$ According to these authors the annealing of a $2 \mathrm{H}$ crystal from about $1450 \mathrm{~K}$ down to room temperature results first in the formation of a $4 H$ polytype, and at lower temperatures a $6 \mathrm{H}$ structure becomes stable followed by the cubic structure which is stable at room temperature. One should note that a $4 H$ polytype consists of " $1_{h} 1_{c}$ " domains and the $6 \mathrm{H}$ structure of " $1_{h} 2_{c}$ " domains; the order of stability of these domains is that given by our empirical stability rules. We do, however, disagree with the Baars and Brandt's reference to the intermediate structures as "polytypes." From the broad peaks of their x-ray diffraction pattern it is clear that the " $1_{h} 1_{c}$ " domains do not constitute a coherent periodic stacking sequence, but rather a large number of such domains stacked along the $c$ axis of the crystal in a macroscopically nonperiodic sequence. This distinction between periodic domain arrangements and nonperiodic arrangement of the same domains is important since it stresses the difference between two thermodynamically similar phenomena differing in their control mechanism, in our case the interaction with a screw dislocation.

The formation of polytypes in materials other than $\mathrm{ZnS}$ may follow a similar mechanism of formation as that operating in $\mathrm{ZnS} ; \mathrm{TiS}_{1.7}$ seems to be such a material. ${ }^{20}$ In general, however, one can expect variations in thermodynamic stability characteristics and control mechanisms to exist between different materials which would show up in the characteristics of the stacking sequences. The polytypes of $\mathrm{SiC}$, the first known polytypic material, have some characteristics common with ZnS. For example, no SiC polytypes were yet found having neighboring hexagonal layers; this combination of layers is also very rare in $\mathrm{ZnS}$. On the other hand, large cubic domains are very common in $\mathrm{ZnS}$ polytypes, while most domains in $\mathrm{SiC}$ polytypes are not larger than $3_{c}$. Neighboring hexagonal layers are very common in $\mathrm{CdI}_{2}$ polytypes, while cubic domains larger than $1_{c}$ are very rare. Single screw dislocations are common in $\mathrm{ZnS}$ crystals, while in $\mathrm{SiC}$ a large number of them is often found in the same crystal.

The role of screw dislocations during crystal growth, enabling rapid growth at low supersaturation by providing a self-perpetuating step, is very common and well established; their role in controlling the structure of the crystal is unique. The formation mechanism of the giant screw dislocations is still unknown and deserves further investigation. As the existence of a number of phases of a compound is common, it is quite plausible that the lack of a periodicity-imposing mechanism is the reason for the rarity of polytypic materials.

One can envision control mechanisms which will support long-range periodicities and will not involve screw dislocations. An intriguing possibility is the epitaxial growth of nonpolytypic materials over polytype substrates. If the material has the appropriate stability characteristics and a good lattice match to the substrate, the necessary periodicity control will be imposed by that 
of the substrate. "Artificial" polytypism may thus be a viable possibility.

\section{SUMMARY AND CONCLUSIONS}

The formation of polytypes is explained in terms of domain stability, which is determined by thermodynamic parameters. While thermodynamic stability may account for the formation of short-period polytypes, a control mechanism must be present in order for long-period polytypes to be formed. The nature of the control mechanism as well as the conditions for domain stability may vary from one material to another. Thermodynamic and dislo- cation theories of polytypism should be considered as supplementary rather than contradictory.

It has been shown in this article that polytypism in vapor-phase-grown $\mathrm{ZnS}$ crystals can be explained in terms of a temperature-dependent stability of cubic and hexagonal domains undergoing post-growth transformations. Screw dislocations are responsible for the periodicity control during these transformations. More experiments are needed in order to establish the stability temperature ranges for the different domains. Analysis of polytypes in crystals annealed at different temperatures is expected to supply this information.
${ }^{1}$ A. Baronnet, Prog. Crystal Growth Charact. 1, 151 (1978).

${ }^{2}$ G. S. Zhdanov, C. R. Acad. Sci. URSS 48, 43 (1945).

${ }^{3}$ B. K. Daniels, Philos. Mag. 14, 487 (1966).

${ }^{4}$ S. Mardix, E. Alexander, O. Brafman, and I. T. Steinberger, Acta Crystallogr. 22, 808 (1967).

${ }^{5}$ S. Mardix, Z. H. Kalman, and I. T. Steinberger, Acta Crystallogr. Sect. A 24, 464 (1968).

6S. Mardix and I. Kiflawi, Cryst. Lattice Defects 1, 129 (1970).

${ }^{7}$ S. Mardix and I. T. Steinberger, J. Appl. Phys. 41, 5339 (1970).

${ }^{8}$ S. Mardix, A. R. Lang, and I. Blech, Philos. Mag. 24, 683 (1971).

${ }^{9}$ S. Mardix, J. Appl. Cryst. 17, 328 (1984).

${ }^{10}$ E. T. Allen and J. L. Crenshaw, Am. J. Sci. 34, 310 (1912).

${ }^{11}$ E. T. Allen and J. C. Crenshaw, Z. Anorg. Chem. 79, 125 (1913).

${ }^{12}$ H. Jagodzinski, Acta Crystallogr. 2, 201 (1949).

${ }^{13}$ G. Shachar, S. Mardix, and I. T. Steinberger, J. Appl. Phys. 39, 2485 (1968).

${ }^{14}$ C. J. Schneer, Acta Crystallogr. 8, 279 (1955).

${ }^{15}$ K. N. Rai and P. Krishna, Indian J. Pure Appl. Phys. 6, 118 (1968).

${ }^{16}$ K. N. Rai and P. Krishna, J. Cryst. Growth 3/4, 741 (1968).

${ }^{17}$ S. Mardix, I. Kiflawi, and Z. H. Kalman, Acta Crystallogr. Sect. B 25, 1586 (1969).

${ }^{18}$ S. Mardix, J. Appl. Crystallogr. 17, 167 (1984).

${ }^{19}$ J. Baars and G. Brandt, J. Phys. Chem. Solids 34, 905 (1973).

20J. J. Legendre, R. Moret, E. Tronc, and H. Huber, J. Appl. Crystallogr. 8, 603 (1975).

${ }^{21}$ C. Frondel and C. Palach, Am. Mineral. 35, 29 (1950).

${ }^{22}$ S. Haussuhl and G. Müller, Mineral. Petrogr. Mitt. 9, 28 (1963).

${ }^{23}$ S. Mardix, O. Brafman, and I. T. Steinberger, Acta Crystallogr. 22, 805 (1967).

${ }^{24}$ H. T. Evans and E. T. McKnight, Am. Mineral. 44, 1210 (1959).
${ }^{25}$ O. Brafman, E. Alexander, and I. T. Steinberger, Acta Crystallogr. 22, 347 (1967).

26I. Kiflawi, Z. H. Kalman, and Y. Sonnenblick, J. Cryst. Growth 34, 145 (1976).

27I. Kiflawi and S. Mardix, Acta Crystallogr. Sect. B 26, 1192 (1970).

${ }^{28}$ I. Kiflawi and S. Mardix, Acta Crystallogr. Sect. B 25, 1195 (1969).

${ }^{29}$ I. Kiflawi, S. Mardix, and I. T. Steinberger, Acta Crystallogr. Sect. B 25, 1581 (1969).

${ }^{30}$ I. Kiflawi, S. Mardix, and Z. H. Kalman, Acta Crystallogr. Sect. B 25, 2413 (1969).

${ }^{31}$ E. Kodera, T. Aikami, and J. Kakinoki, Acta Crystallogr. Sect. A 28, Suppl. 4, S119 (1972).

${ }^{32}$ S. Kume, E. Kodera, T. Aikami, and J. Kakinoki, J. Phys. Soc. Jpn. 32, 288 (1972).

${ }^{33}$ S. Mardix and O. Brafman, Acta Crystallogr. 23, 501 (1967).

${ }^{34}$ S. Saliba and S. Mardix, Acta Crystallogr. Sect. A 39, 933 (1983).

${ }^{35}$ V. Medizadeh and S. Mardix, Acta Crystallogr. (to be published).

${ }^{36}$ S. Mardix and O. Brafman, Acta Crystallogr. Sect. B 24, 258 (1968).

${ }^{37}$ I. Kiflawi, S. Mardix, and I. T. Steinberger, Acta Crystallogr. Sect. B 27, 378 (1971).

${ }^{38}$ I. Kiflawi and S. Mardix, Acta Crystallogr. Sect. B 25, 2415 (1969).

${ }^{39}$ Y. Takano and T. Nishida, Acta Crystallogr. Sect. A 28, Suppl. 4, S119 (1972).

${ }^{40}$ Y. Sonnenblick and I. Kiflawi, Isr. J. Chem. 10, 7 (1972).

${ }^{41}$ I. T. Steinberger, E. Alexander, Y. Brada, Z. H. Kalman, I. Kiflawi, and S. Mardix, J. Cryst. Growth 13/14, 285 (1972).

${ }^{42}$ I. Kiflawi, Z. H. Kalman, S. Mardix, and I. T. Steinberger, Acta Crystallogr. Sect. B 28, 2110 (1972). 